\title{
Technical Note: Multispectral lidar time series of pine canopy chlorophyll content
}

\author{
T. Hakala ${ }^{1}$, O. Nevalainen ${ }^{1}$, S. Kaasalainen ${ }^{1}$, and R. Mäkipää ${ }^{2}$ \\ ${ }^{1}$ Finnish Geospatial Research Institute (FGI), Masala, Finland \\ ${ }^{2}$ Finnish Forest Research Institute (METLA), Vantaa, Finland \\ Correspondence to: T. Hakala (teemu.hakala@nls.fi)
}

Received: 9 June 2014 - Published in Biogeosciences Discuss.: 23 October 2014

Revised: 30 January 2015 - Accepted: 16 February 2015 - Published: 12 March 2015

\begin{abstract}
We present an empirical application of multispectral laser scanning for monitoring the seasonal and spatial changes in pine chlorophyll $(a+b)$ content and upscaling the accurate leaf-level chlorophyll measurements into branch and tree level. The results show the capability of the new instrument for monitoring the changes in the shape and physiology of tree canopy: the spectral indices retrieved from the multispectral point cloud agree with laboratory measurements of the chlorophyll $a$ and $b$ content. The approach opens new prospects for replacing destructive and labourintensive manual sampling with remote observations of tree physiology.
\end{abstract}

\section{Introduction}

The photosynthetic activity of leaves within a tree canopy is an indicator of tree health. Vigorous trees with high foliar biomass and chlorophyll content have high carbon assimilation capacity. Stress in vegetation has been shown to induce changes in the photosynthetically active pigments such as chlorophyll $a$ and $b$. Therefore, the leaf chlorophyll content is an important indicator of the photosynthetic capacity as well as tree productivity and stress (Coops et al., 2003; Lausch et al., 2013).

The leaf properties and the distribution of chlorophylls and nutrients within a canopy vary as a function of time and space, and depending on the resource availability (Wang and Schjoerring, 2012; Peltoniemi et al., 2012). Plant phenology and seasonal chlorophyll content cycle are correlated to the $\mathrm{CO}_{2}$ flux. For monitoring these seasonal variations, methods are needed for accurate and non-destructive chlorophyll es- timation, both at the leaf and canopy level (e.g. Gond et al., 1999). Chlorophyll estimation with spectral remote sensing has been implemented increasingly in a number of studies (e.g. Coops et al., 2003; Lausch et al., 2013), but improved resolution and more accurate 3 -D position for the spectra are still being called for to extend the accurate leaf-level measurement into canopy and stand level (cf. Gaulton et al., 2013). To investigate the spatial variation of the photosynthetic capacity and self-shading of photosynthetically active tissue, the canopy and branch structure must also be included in the measurement.

One way to provide simultaneous structural and spectral information is lidar combined with hyperspectral passive sensing (e.g. Thomas et al., 2006; Asner et al., 2007; Jones et al., 2010). New applications using multi- or hyperspectral laser scanning have become more common recently. Hancock et al. (2012) demonstrated the potential of dual-wavelength, large-footprint, spaceborne lidar to separate ground and canopy returns using the extra information contained in a spectral ratio to complement the canopy height from laser scanning. Three-dimensional (3-D) distributions of vegetation biochemical properties were measured with spectral indices developed for the Salford Advanced Laser Canopy Analyser (SALCA), which is also a dual-wavelength lidar (Gaulton et al., 2013). A similar approach was used in the Dual-Wavelength Echidna Lidar (DWEL) (Douglas et al., 2012). A multispectral canopy lidar has also been introduced for simultaneous retrieval of vegetation structure and spectral indices (Woodhouse et al., 2011). In this approach, a tunable laser operating at four wavelengths was used.

In this technical note, an application of the recently developed hyperspectral lidar instrument (HSL) (Hakala et al., 
2012) is presented for monitoring the seasonal and spatial changes in pine total chlorophyll content (chlorophyll $a+b$ ). As a non-destructive method, the capability of the instrument to upscale the accurate leaf-level chlorophyll content measurements into branch and tree level has been investigated and validated with chemical analysis of chlorophyll content. In our recent study (Nevalainen et al., 2014) we determined three spectral indices having good correlation with Scots pine shoot chlorophyll concentration. These indices were also used in this study

\section{Materials and methods}

The HSL is a prototype laser scanning instrument (Hakala et al., 2012) utilizing a supercontinuum laser. White laser (420-1680 nm) pulses are transmitted to a target and the distances of reflected echoes are determined from time of flight. A spectrograph and an avalanche photodiode (APD) array connected to a high-speed digitizer are used to determine the spectrum of each returning echo by measuring the intensity of the echo at multiple wavelengths. Also, the intensity of each transmitted laser pulse is measured and used to normalize the echo intensity. Current prototype configuration uses a 16-element APD array and an 8-channel digitizer, enabling us to measure at 8 wavelength bands: $545,641,675,711$, $742,778,978$ and $1292 \mathrm{~nm}$, with full width at half maximum of $20 \mathrm{~nm}$. Before the target is measured, a reference target with known reflectance (Spectralon) is measured at distance intervals of approximately $30 \mathrm{~cm}$, and these data are used to calibrate the reflectance over the whole measurement range. Additionally, the Spectralon is placed in the scanned area during the actual measurement to validate the calibration. The instrument and data processing are presented in more detail in Hakala et al. (2012).

A Scots pine (Pinus sylvestris L.) was scanned five times during the 2013 growth season. The tree was approximately 13 years old, $5.5 \mathrm{~m}$ high and was growing in a small forest stand near the institute building. The HSL was mounted on a portable cart, and the tree was scanned from two directions. The scans were co-registered using white spherical reference targets placed on fixed locations on the target area. The distance between the scanner and the tree was approximately $5 \mathrm{~m}$. The tree was scanned with $0.1^{\circ}$ horizontal and approximately $0.02^{\circ}$ vertical resolution and the resulting point clouds contained $200000-470000$ echoes from the tree. The beam diameter at the target was approximately $5 \mathrm{~mm}$.

Needle samples were taken immediately after the scan for laboratory analysis. Six branches were selected and the samples were taken from these branches according to needle cohorts (current year needles, and 1-, 2- and 3-year-old needles). Two needle pairs were taken from each cohort of each selected branch. Analysis of the chlorophyll contents followed the protocol described in Wellburn (1994) for extrac- tion with dimethyl sulfoxide (DMSO). After extraction, the chlorophyll concentrations were determined from solvents spectrophotometrically using wavelengths of 480.0, 649.1 and $665.1 \mathrm{~nm}$ (resolution $0.1-0.5 \mathrm{~nm}$ ).

Two of the six sampled branches were clearly identifiable from the multispectral point cloud, having enough point density and long enough growth of the branch. Previous-year cohorts were selected for further analysis, since they had needles present during all measurements. Therefore the following analysis is performed for two cohorts and five measurement dates. The parts of the point cloud containing the selected cohorts were isolated in post processing. Three spectral indices were tested for determining chlorophyll content of the needles. Since it was not possible to tune all required wavelengths to optimal positions for every index, we used the nearest available band.

The modified chlorophyll absorption ratio index (Eq. 1) using reflectance at 705 and $750 \mathrm{~nm}$ (referred here as MCARI750) was first presented by Wu et al. (2008). Contrary to the original MCARI (Daughtry et al., 2000), MCARI750 uses reflectance at 705 and $750 \mathrm{~nm}$, which have shown better sensitivity to high chlorophyll contents (Wu et al., 2008). MCARI was designed to measure the depth of the maximum chlorophyll absorption at $670 \mathrm{~nm}$ relative to green reflectance peak at 550 and reflectance at $700 \mathrm{~nm}$, at canopy scale (Daughtry et al., 2000).

$$
\begin{aligned}
& \operatorname{MCARI750}=\left[\left(R_{750}-R_{705}\right)-0.2 \times\left(R_{750}-R_{550}\right)\right] \\
& \times\left(R_{750} / R_{705}\right)
\end{aligned}
$$

The goal of the modified simple ratio (MSR, Eq. 2), developed by Chen (1996), is to achieve low noise effect and good linearity to vegetation biophysical parameters. MSR has been used to estimate chlorophyll and leaf area index (LAI) at canopy scale. Wu et al. (2008) also developed MSR using reflectance at 705 and $750 \mathrm{~nm}$, referred to here as MSR2.

$\operatorname{MSR} 2=\frac{R_{750} / R_{705}-1}{\sqrt{R_{750} / R_{705}+1}}$

The simple ratio (SR, Eq. 3) indices directly compare the reflectance and absorbance peaks of chlorophyll pigments, which make them sensitive to changes in chlorophyll content (Wu et al., 2008). A variety of wavelength combinations are used with simple ratio indices, but the one selected for this study is SR6 (Zarco-Tejada et al., 2001).

$\mathrm{SR} 6=\frac{R_{750}}{R_{710}}$

Additionally, normalized difference vegetation index (NDVI, Eq. 4) (Rouse et al., 1973) was used to separate needles from branches. NDVI is the most widely used vegetation index. It is based on the contrast between high absorption at red and high reflectance at near infrared (NIR). NDVI was developed for the canopy scale, and it has previously been used for both 
chlorophyll and LAI estimation.

$\mathrm{NDVI}=\frac{R_{800}-R_{670}}{R_{800}+R_{670}}$

As the channels of the prototype lidar instrument are limited to eight separate spectral bands, these indices had to be used with the closest available spectral band (Table 1).

The limitation of empirical vegetation indices estimating chlorophyll content is that they are also affected by the canopy structural properties. In addition, they can be affected by the internal structure, size, surface and shape of leaves and can thus be species-specific, requiring calibration when applied to specific species (Zhang et al., 2008).

The benefit of active measurement systems, such as HSL, is that they measure backscattered signal that has the potential to eliminate many of the multiple scattering and geometric viewing effects caused by the canopy structure (Gaulton et al., 2013; Morsdorf et al., 2009). The major factors affecting the backscattered signal are the local incidence angle of the target and the area of effective backscattering surface (Gaulton et al., 2013). These factors are also present in this study, as one $5 \mathrm{~mm}$ footprint may include one or several needles with varying incidence angles. However, the influence of these factors is similar with different wavelengths measured at the same optical path. Thus, by calculating spectral ratios (i.e. vegetation indices), the influence of the incidence angle and target area can be reduced (Eitel et al., 2011; Gaulton et al., 2013).

\section{Results}

A 3-D point cloud of the tree and changes in structure (such as the growth of new shoots) from May to November can be observed in Fig. 1, where no spectral information is used. The changes in the structure of one branch are visible in the coloured point clouds in Fig. 2, where we plot the NDVI time series of the pine branch from 15 May to 6 November 2013. The outbreak and growth of new shoots (May-June 2013) can be observed, as well as the year 2 cohorts defoliating (September-October 2013) and falling off completely (November 2013).

To validate the capability of the HSL to estimate the chlorophyll content using spectral indices, we compared the lidar data with laboratory analysis over the growing season. We present data for two branch cohorts, denoted M2_1 and M3_1 (1-year-old part of M2 and M3), which were best visible in the multispectral point clouds. The trends in the chlorophyll content and the indices MCARI750, MSR2 and SR6 from HSL data are well reproduced for the individual branches (Figs. 3-5). For all three indices, the sample branch M2_1 was best correlated with the laboratory measurements with $R^{2}$ values of 0.8-0.9. The $R^{2}$ for MCARI750 and MSR2 for M3_1 was 0.7, whereas SR6 performed worse for M3_1 $\left(R^{2} 0.4\right)$. When the data from M2_1 and M3_1
Table 1. The available channel wavelengths $(\mathrm{nm})$ and the nominal wavelengths $(\mathrm{nm})$ of the spectral indices. The closest available channel was used.

\begin{tabular}{lrrrr}
\hline Channel & MCARI750 & MSR2 & SR6 & NDVI \\
\hline 545 & 550 & & & \\
641 & & & & \\
675 & & & & 670 \\
711 & 705 & 705 & 710 & \\
742 & 750 & 750 & 750 & \\
778 & & & & 800 \\
978 & & & & \\
1292 & & & & \\
\hline
\end{tabular}

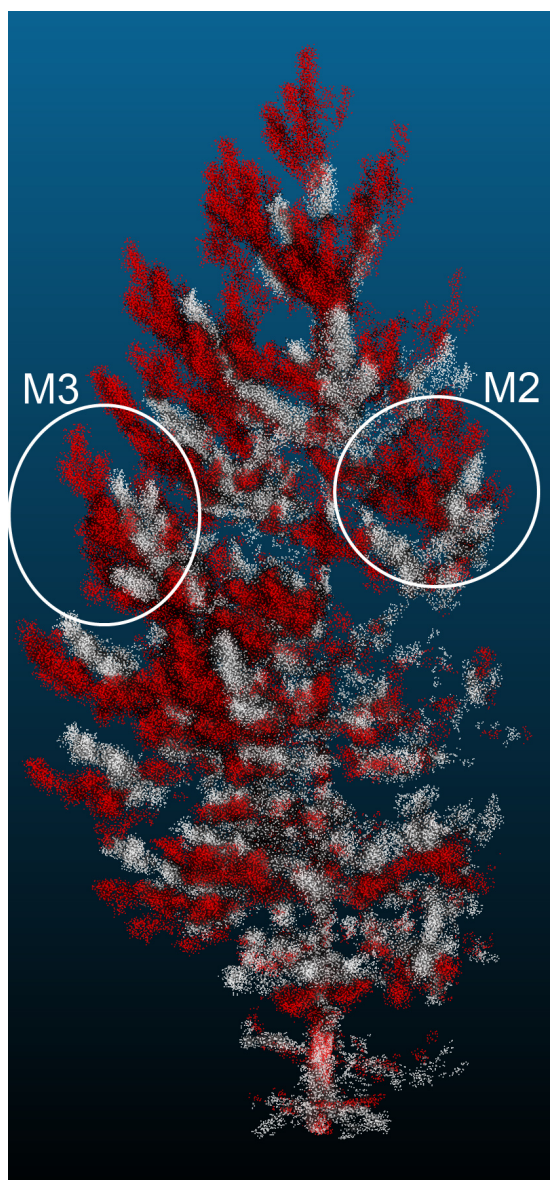

Figure 1. Co-registered point clouds from the 15 May 2013 scan (grey) and the 11 June 2013 scan (red). Growth of the tree is visible and also some movement of the branches can be observed. The height of the tree is approximately $5.5 \mathrm{~m}$.

were combined for regression, all indices correlated with the chlorophyll contents measured in the laboratory (Fig. 6). The results were worse for indices averaged over the entire tree point cloud (the right column in Figs. 3-5), compared with the average of all year 1 needles measured in the laboratory. This is very likely a result of the variation of the physio- 

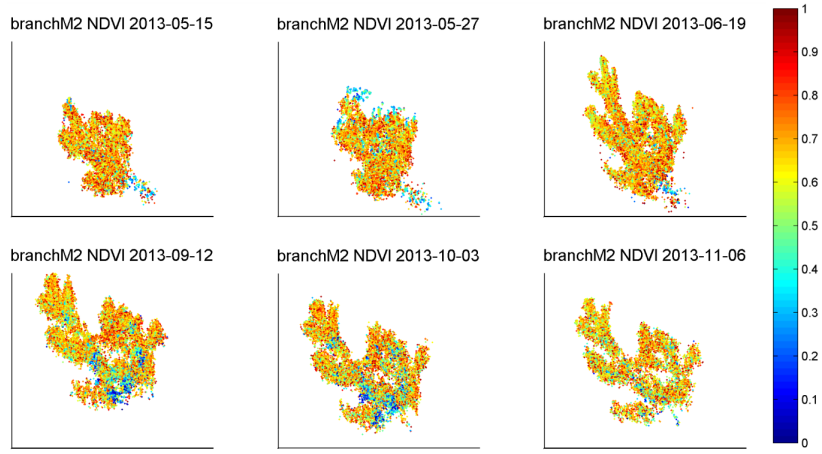

Figure 2. NDVI (see the colour bar for values) point clouds of a sample branch M2. The growth of new needles (starting 27 May), already clearly visible new branch tips 19 June, fully grown new needles 12 September and dying and falloff of old needles (low NDVI in 12 September and 03 October) are visible in the data measured at different times. The measurement dates are shown in the plot titles.
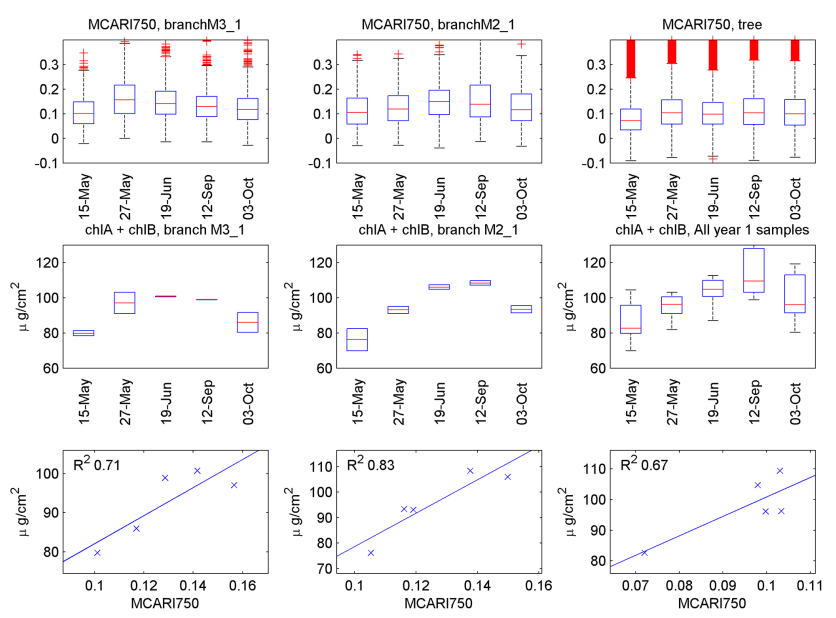

Figure 3. Top row: distribution of MCARI750 spectral index during separate scans - the central mark is the median, the edges of the box are the 25th and 75th percentiles, and the whiskers extend to the most extreme data points not considered outliers. Middle row: laboratory measurements of chlorophyll $a+b$ content per needle surface area. Bottom row: correlation of the spectral index and laboratory measurement. Subplot columns left to right: sample branch 3 , year 1; sample branch 2, year 1; and spectral index of whole tree and laboratory measurements of all year 1 samples.

logical conditions between different branches, which is more pronounced when the sampling is carried out over the entire tree (i.e. the point cloud) rather than just a few needle samples (as in the laboratory experiment). All in all, the analysis of branch cohorts shows that the spatial distribution of the lidar-based spectral indices describes the chlorophyll content within the branch, although more measurements are needed to better validate the results.

In Figs. 3-5, branch M2_1 and M3_1 laboratory measurements consist of two separate needles only. More sampling
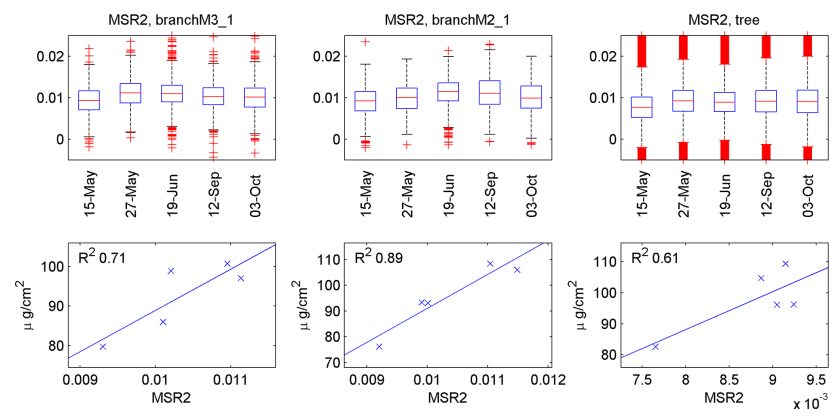

Figure 4. Same as previous figure (top and bottom rows; laboratory data are the same as in previous figure) but this time using the MSR2 spectral index.
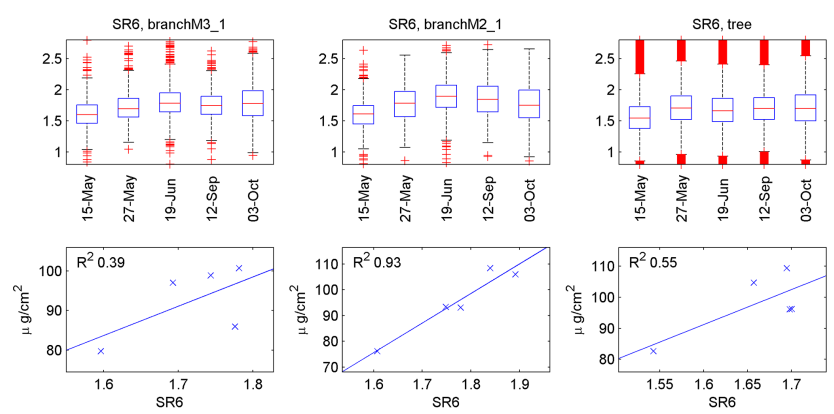

Figure 5. Same as previous figure but this time using the SR6 spectral index.

should have been performed; however, the number of needles in each branch cohort is limited and the tree had to be sampled several times during the year (this emphasizes the need for non-destructive methods). The number of laser echoes from year 0 and 2 were highly variable; in the spring lidar point clouds, the year 0 growths were very small, providing very few echoes. The year 2 and older cohorts started dropping needles before September measurement, thus reducing the number of echoes during autumn compared to spring. Therefore we only used year 1 laboratory measurement of needles in plots 3-5 for whole tree (right column), since the weight of the year 0 and 2 laboratory measurements would have been higher compared to the lidar point cloud (lidar point density variable and laboratory sample number constant). Some lidar echoes still originate from the year 0 and 2 needles, reducing the overall correlation between laboratory and lidar data for the whole tree.

The changes in the structure of the tree are visible in Fig. 1. The fact that the structure of the tree can be retrieved from laser scanner point clouds has been shown before in numerous studies (see Kaasalainen et al., 2014, and references therein). We have also shown in our previous study that the tree structure and its changes can be quantified from laser scanner point clouds using quantitative structure modelling (QSM) designed to retrieve tree branching structures (Raumonen et al., 2013; Kaasalainen et al., 2014). As the scope 

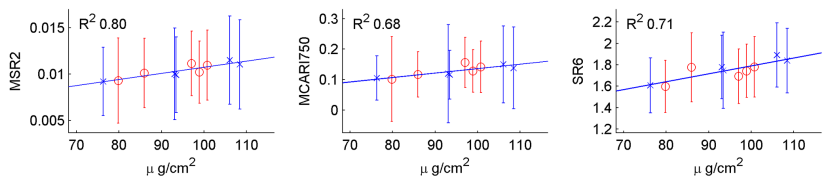

Figure 6. Correlation of spectral index and laboratory measurement for combined M2_1 and M3_1 data. Left: MSR2; middle: MCARI750; right: SR6. Blue x: M3_1; red circle: M2_1.

of this note was to show the added value of spectral data in the chlorophyll distribution monitoring, the changes in tree structure will be an object of our future study.

\section{Discussion and conclusions}

We have shown that the multispectral lidar provides an empirical approach for efficiently mapping the spatial distributions of tree physiological parameters that are correlated with reflectance of the foliage (such as chlorophyll $a$ and $b$ ). Because the method of measurement is non-destructive, it can be repeated for the same target to produce time series of important tree functions, such as moisture condition, photosynthetic capacity, or physiological status.

We demonstrated that the seasonal changes in the structure and physiology of tree canopy, needles and branches are visible in 3-D; parameters affecting tree physiology can be quantified with spectral indices and linked to a specific location in the tree canopy using the multispectral point cloud. We validated the method with reference measurements of chlorophyll $a$ and $b$ concentration in a laboratory. According to our results, multispectral lidar can be used for the monitoring of the chlorophyll content, but similarly, the approach has potential in the monitoring of the water, carotenoid or lignin content, which all affect reflectance of the foliage (Austin and Ballare, 2010).

Although the influence of multiple scattering effects caused by canopy structure can be reduced using multispectral lidar and ratios of backscattered reflectance, it is not completely removed. Further study would be required to produce a physically based model that would properly account for the multiple scattering of needles within a single laser footprint and its effect to the measured backscattered reflectance. In addition, some of the limitations of vegetation indices in chlorophyll estimation, such as robustness and portability to different measurement configuration and wavelengths, might be overcome by using inversion of radiative transfer models, such as the LIBERTY (Leaf Incorporating Biochemistry Exhibiting Reflectance and Transmittance Yields) (Dawson et al., 1998), which is specifically developed for needles, , or by utilizing PROSPECT (leaf optical properties) model to optimize spectral indices (Féret et al., 2011).

The tree was scanned from two directions only. Increasing the number of scans from different directions around the tree will improve the results by increasing the point coverage. This will require some instrument development to allow for more efficient field use. Increasing the point density is also an important object of instrument improvement. However, the prototype instrument was capable of showing the potential of 3-D spectral measurements.

A major factor causing error and uncertainty in this research was the use of the nearest possible channel in vegetation index calculation instead of the band that the index was designed to use. This affects the performance of the vegetation indices, especially with indices requiring channels at the red edge, where even a small shift in channel wavelength causes a large change in reflectance. However, this was not considered to be major problem, as the aim of this study was to test the ability of our HSL instrument in chlorophyll estimation and not to optimize the performance of the indices.

Further work is needed to find the best spectral indices for different applications (e.g. monitoring the 3-D effects of drought or limited amount of light on the physiology of a tree) and then optimize the spectral channels to match with these indices. This will improve the precision of the results. Increasing the number of spectral channels would also improve the channel optimization and efficiency. Once the approach is well established and calibrated, it has potential for replacing a number of laborious and destructive manual experiments, and hence providing a new tool for remote observations of tree physiology. Although the first results show the potential of the approach, further studies on backscatter of the supercontinuum laser from the canopy are needed to establish the method physically.

Acknowledgements. This study was funded by the Academy of Finland research projects "New techniques in active remote sensing: hyperspectral laser in environmental change detection" and "Mobile hyperspectral laser remote sensing".

Edited by: A. MacArthur

\section{References}

Asner, G. P., Knapp, D. E., Kennedy-Bowdoin, T., Jones, M. O., Martin, R. E., Boardman, J., and Field, C. B: Carnegie Airborne Observatory: in-flight fusion of hyperspectral imaging and waveform light detection and ranging for three-dimensional studies of ecosystems, J. Appl. Remote Sens., 1, 013536, doi:10.1117/1.2794018, 2007.

Austin, A. T. and Ballaré, C. S.: Dual role of lignin in plant litter decomposition in terrestrial ecosystems, P. Natl. Acad. Sci. USA, 107, 4618-4622, doi:10.1073/pnas.0909396107, 2010.

Chen, J.: Evaluation of vegetation indices and modified simple ratio for boreal applications, Can. J. Remote Sens., 22, 229-242, 1996.

Coops, N. C., Stone, C., Culvenor, D. S., Chisholm, L. A., and Merton, R. N.: Chlorophyll content in eucalypt vegetation at the leaf and canopy scales as derived from high resolution spectral data, Tree Physiol., 23, 23-31, doi:10.1093/treephys/23.1.23, 2003. 
Daughtry, C.: Estimating Corn Leaf Chlorophyll Concentration from Leaf and Canopy Reflectance, Remote Sens. Environ., 74, 229-239, doi:10.1016/S0034-4257(00)00113-9, 2000.

Dawson, T. P., Curran, P. J., and Plummer, S. E.: LIBERTY - modeling the effects of leaf biochemical concentration on reflectance spectra, Remote Sens. Environ., 65, 50-60, 1998.

Douglas, E. S., Strahler, A., Martel, J., Cook, T., Mendillo, C., Marshall, R., Chakrabarti, S., Schaaf, C., Woodcock, C., Li, Z., Yang, X., Culvenor, D., Jupp, D., Newnham, G., and Lovell, J.: DWEL: A Dual-Wavelength Echidna Lidar for ground-based forest scanning, 4998-5001, Int. Geosci. Remote Se., 2012.

Eitel, J. U. H., Vierling, L. A., Long, D. S., and Hunt, E. R.: Early season remote sensing of wheat nitrogen status using a green scanning laser, Agr. Forest Meteorol., 151, 1338-1345, doi:10.1016/j.agrformet.2011.05.015, 2011.

Féret, J. B., François, C., Gitelson, A., Asner, G. P., Barry, K. M., Panigada, C., Richardson, A. D., and Jacquemoud, S.: Optimizing spectral indices and chemometric analysis of leaf chemical properties using radiative transfer modeling, Remote Sens. Environ., 115, 2742-2750, doi:10.1016/j.rse.2011.06.016, 2011.

Gaulton, R., Danson, F. M., Ramirez, F. A., and Gunawan, O.: The potential of dual-wavelength laser scanning for estimating vegetation moisture content, Remote Sens. Environ., 132, 32-39, doi:10.1016/j.rse.2013.01.001, 2013.

Gond, V., de Pury, D. G. G., Veroustraete, F., and Ceulemans, R.: Seasonal variations in leaf area index, leaf chlorophyll, and water content; scaling-up to estimate fAPAR and carbon balance in a multilayer, multispecies temperate forest, Tree Physiol., 19, 673679, doi:10.1093/treephys/19.10.673, 1999.

Hakala, T., Suomalainen, J., Kaasalainen, S., and Chen, Y.: Full waveform hyperspectral LiDAR for terrestrial laser scanning, Opt. Express, 20, 7119, doi:10.1364/OE.20.007119, 2012.

Hancock, S., Lewis, P., Foster, M., Disney, M., and Muller, J.P.: Measuring forests with dual wavelength lidar: A simulation study over topography, Agr. Forest Meteorol., 161, 123-133, doi:10.1016/j.agrformet.2012.03.014, 2012.

Jones, T. G., Coops, N. C., and Sharma, T.: Assessing the utility of airborne hyperspectral and LiDAR data for species distribution mapping in the coastal Pacific Northwest, Canada, Remote Sens. Environ., 114, 2841-2852, doi:10.1016/j.rse.2010.07.002, 2010.

Kaasalainen, S., Krooks, A., Liski, J., Raumonen, P., Kaartinen, H., Kaasalainen, M., Puttonen, E., Anttila, K., and Mäkipää, R.: Change Detection of Tree Biomass with Terrestrial Laser Scanning and Quantitative Structure Modelling, Remote Sens., 6, 3906-3922, doi:10.3390/rs6053906, 2014.

Lausch, A., Heurich, M., Gordalla, D., Dobner, H.-J., GwillymMargianto, S., and Salbach, C.: Forecasting potential bark beetle outbreaks based on spruce forest vitality using hyperspectral remote-sensing techniques at different scales, Forest Ecol. Manag., 308, 76-89, doi:10.1016/j.foreco.2013.07.043, 2013.
Morsdorf, F., Nichol, C., Malthus, T., and Woodhouse, I. H.: Assessing forest structural and physiological information content of multi-spectral LiDAR waveforms by radiative transfer modelling, Remote Sens. Environ., 113, 2152-2163, doi:10.1016/j.rse.2009.05.019, 2009.

Nevalainen, O., Hakala, T., Suomalainen, J., Mäkipää, R., Peltoniemi, M., Krooks, A., and Kaasalainen, S.: Fast and nondestructive method for leaf level chlorophyll estimation using hyperspectral LiDAR, Agr. Forest Meteorol., 198-199, 250-258, doi:10.1016/j.agrformet.2014.08.018, 2014.

Peltoniemi, M. S., Duursma, R. A., and Medlyn, B. E.: Cooptimal distribution of leaf nitrogen and hydraulic conductance in plant canopies, Tree Physiol., 32, 510-519, doi:10.1093/treephys/tps023, 2012.

Raumonen, P., Kaasalainen, M., Åkerblom, M., Kaasalainen, S., Kaartinen, H., Vastaranta, M., Holopainen, M., Disney, M., and Lewis, P.: Fast Automatic Precision Tree Models from Terrestrial Laser Scanner Data, Remote Sens., 5, 491-520, doi:10.3390/rs5020491, 2013.

Rouse, J. W., Haas, R. H., Schell, J. A., and Deering, W. D.: Monitoring vegetation systems in the Great Plains with ERTS, Third ERTS Symposium, NASA SP-351, 309-317, 1973

Thomas, V., Finch, D. A., McCaughey, J. H., Noland, T., Rich, L., and Treitz, P.: Spatial modelling of the fraction of photosynthetically active radiation absorbed by a boreal mixedwood forest using a lidar-hyperspectral approach, Agr. Forest Meteorol., 140, 287-307, doi:10.1016/j.agrformet.2006.04.008, 2006.

Wang, L. and Schjoerring, J. K.: Seasonal variation in nitrogen pools and ${ }^{15} \mathrm{~N} /{ }^{13} \mathrm{C}$ natural abundances in different tissues of grassland plants, Biogeosciences, 9, 1583-1595, doi:10.5194/bg9-1583-2012, 2012.

Wellburn, A. R.: The Spectral Determination of Chlorophylls $a$ and $b$, as well as Total Carotenoids, Using Various Solvents with Spectrophotometers of Different Resolution, J. Plant Physiol., 144, 307-313, doi:10.1016/S0176-1617(11)81192-2, 1994.

Woodhouse, I. H., Nichol, C., Sinclair, P., Jack, J., Morsdorf, F., Malthus, T. J., and Patenaude, G.: A Multispectral Canopy LiDAR Demonstrator Project, IEEE Geosci. Remote Sens. Lett., 8 , 839-843, doi:10.1109/LGRS.2011.2113312, 2011.

Wu, C., Niu, Z., Tang, Q., and Huang, W.: Estimating chlorophyll content from hyperspectral vegetation indices: Modeling and validation, Agr. Forest Meteorol., 148, 1230-1241, doi:10.1016/j.agrformet.2008.03.005, 2008.

Zarco-Tejada, P. J., Miller, J. R., Noland, T. L., Mohammed, G. H., and Sampson, P. H.: Scaling-up and model inversion methods with narrowband optical indices for chlorophyll content estimation in closed forest canopies with hyperspectral data, IEEE T. Geosci. Remote, 39, 1491-1507, doi:10.1109/36.934080, 2001.

Zhang, Y., Chen, J., Miller, J., and Noland, T.: Leaf chlorophyll content retrieval from airborne hyperspectral remote sensing imagery, Remote Sens. Environ., 112, 3234-3247, doi:10.1016/j.rse.2008.04.005, 2008. 